Submitted to 1995 Particle Accelerator Conference, Dallas, Texas, May 1-5, 1995.

\title{
ORBIT STABILITY IMPROVEMENT AT THE NSLS X-RAY RING *
}

\author{
J. Safranek, O. Singh, and L. Solomon \\ National Synchrotron Light Source, Brookhaven National Laboratory, Upton, NY 11973
}

Abstract

We describe recent improvements in both the short and long-term orbit stability at the NSLS X-Ray Ring. The short-term stability has been improved by increasing the gain and the bandwidth of the global harmonic feedback systems. The long-term horizontal orbit drift over the course of a fill has been reduced by including the rffrequency in the orbit feedback. Work is in progress to further reduce orbit drift by compensating for thermally induced mechanical motions of the beam position monitors (BPMs).

\section{INTRODUCTION}

At the NSLS storage rings, the harmonic based analog global feedback system has provided excellent orbit stability [1]. For most users, the level of this beam stability (short and long-term) has been satisfactory, however, further stabilization is desirable. During the Fall of 1994, the gain and bandwidth of the global feedback systems were increased resulting in significantly reduced short-term orbit fluctuations. The long-term drift in the horizontal orbit has been reduced by up to a factor of 3.6 by varying the rf frequency to eliminate orbit drift proportional to the dispersion.

With the improvements in the global feedback and the addition of the rf frequency feedback, the present limit in orbit stability is the accuracy of the BPMs. The primary limitation in reading the horizontal orbit is the motion of the vacuum chamber at the BPMs due to synchrotron radiation heating. In this paper we describe work to characterize this vacuum chamber motion so that we can compensate for it in the orbit feedbacks. The vacuum chamber moves very little in the vertical plane, so the orbit stability vertically is limited by the variation in BPM processing electronics with stored current. The present BPM electronics are stable to about 10 to. $20 \mu m$ over the range of operating currents ( 110 to $250 \mathrm{~mA}$ ). New BPM electronics with dynamic stability better than $5 \mu m$ have been developed recently [2], and we plan to implement these soon for further stabilization of the vertical orbit.

Concurrently, we are developing a new digital orbit feedback system to achieve even better performance [3].

\section{IMPROVED GLOBAL FEEDBACK}

By increasing the gain and bandwidth of the servo loops for feedback harmonics, the feedback system performance has been enhanced. The improvement can be described by analyzing the overall open-loop-gain and phase responses.

"Work performed under the auspices of the U.S. Department of Energy
Figure 1 shows the gain and phase responses for both the old and new systems. The gain response for the new system (drawn with the solid line) shows a gain increase of $9 \mathrm{db}$ (factor of 3 ), as compared to the old system (drawn with a dotted line). This indicates that the noise at low frequencies was reduced by an additional factor of 3 when the new system was implemented. The bandwidth is defined as the frequency where gain curve crosses $0 \mathrm{db}$. The gain curves show that the bandwidth has been increased from $70 \mathrm{~Hz}$ to $200 \mathrm{~Hz}$, so the beam noise in this frequency range is reduced. The phase response (shown by solid line in the lower plot) has been modified so that the 45 degree phase margin extends to $200 \mathrm{~Hz}$ to meet the stability requirement for the feedback system.

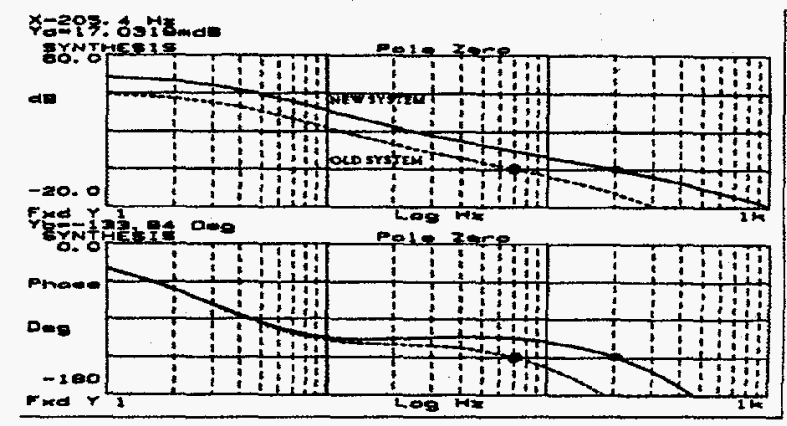

Figure. 1. Gain and phase response of the old global feedback compared to the improved high-gain, broadbandwidth global feedback.

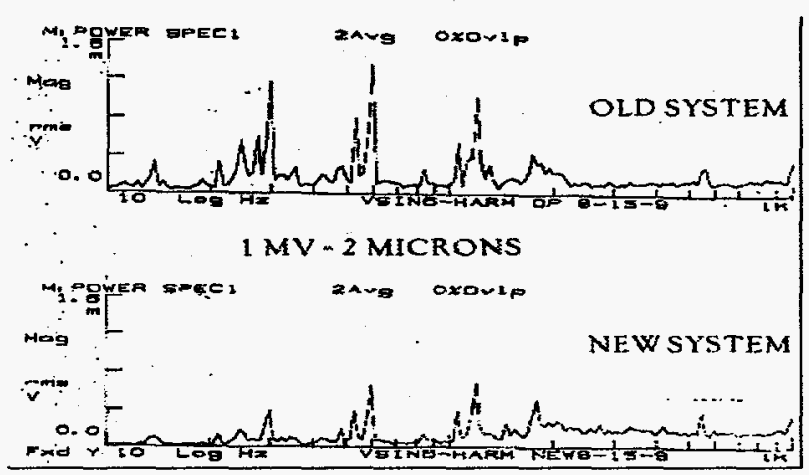

Figure. 2. A spectral analysis of a sixth spatial harmonic of the vertical orbit. The new global feedback system shows improved reduction in orbit fluctuations up to $200 \mathrm{~Hz}$.

Figure 2 shows the frequency spectrums for a 6 th spatial orbit harmonic in vertical plane. The top trace shows the beam motion harmonic when old feedback was in op- 
eration; the bottom trace shows the beam motion harmonic when the new feedback system was in operation (the present operating conditions). The frequency peaks from 1 to $120 \mathrm{~Hz}$ have been reduced. The improvement factor at 3 frequencies are listed in table 1.

Table 1. Improvement seen at different frequencies in orbit noise reduction with the new increased-bandwidth global feedback.

\begin{tabular}{|c|c|}
\hline Frequency & $\begin{array}{c}\text { Approximate } \\
\text { Improvement factor }\end{array}$ \\
\hline $30 \mathrm{~Hz}$ & 3 \\
$60 \mathrm{~Hz}$ & 2 \\
$120 \mathrm{~Hz}$ & 1.5 \\
\hline
\end{tabular}

\section{RF FREQUENCY FEEDBACK [4]}

The improved gain and bandwidth of the global feedback system primarily reduced orbit motion on time scales less than one second. We have also made recent progress toward reducing orbit drift on time scales of hours. We noticed that during many fills, much of the horizontal orbit drift had a shape similar to the X-Ray Ring dispersion. For example, the solid line in figure 3 has the largest orbit distortions in the eight dispersion straight sections. This means that we can eliminate this orbit distortion by varying the of frequency. We have tested a program that changes the rf frequency every five seconds to remove any dispersion-like orbit shift. The change in rf frequency, $\Delta f_{r f}$ is calculated as follows:

$$
\Delta f_{r f}=\frac{\left(\mathbf{x}-\mathbf{x}_{0}\right) \cdot \frac{d \mathbf{x}}{d f_{r f}}}{\frac{d \mathbf{X}}{d f_{r f}} \cdot \frac{d \mathbf{X}}{d f_{r f}}}
$$

where $\mathbf{x}$ is the measured orbit, $\mathbf{x}_{0}$ is the orbit where the synchrotron radiation users want the beam to stay, and $\frac{d \mathbf{X}}{d f_{r f}}$ is the measured change in orbit with rf frequency (measured with the other global and local orbit feedbacks running). The measured orbit, $\mathbf{x}$, is actually the average of 1280 orbits measured over five seconds. Each five seconds, only $1 / 5$ th of the calculated change in $f_{r f}$ is implemented. With all this averaging, the implemented change in the rf frequency for each 5 second step is rarely larger than one bit which is $0.1 \mathrm{~Hz}$ and gives about $0.2 \mu \mathrm{m}$ change in orbit at the beamline source points. Therefore no appreciable noise is added to the orbit while the long-term drift proportional to the dispersion is eliminated. Figure 3 shows the results of testing the $f_{r f}$ feedback during a studies shift. The rms drift in horizontal orbit was reduced by a factor of 3.6 .

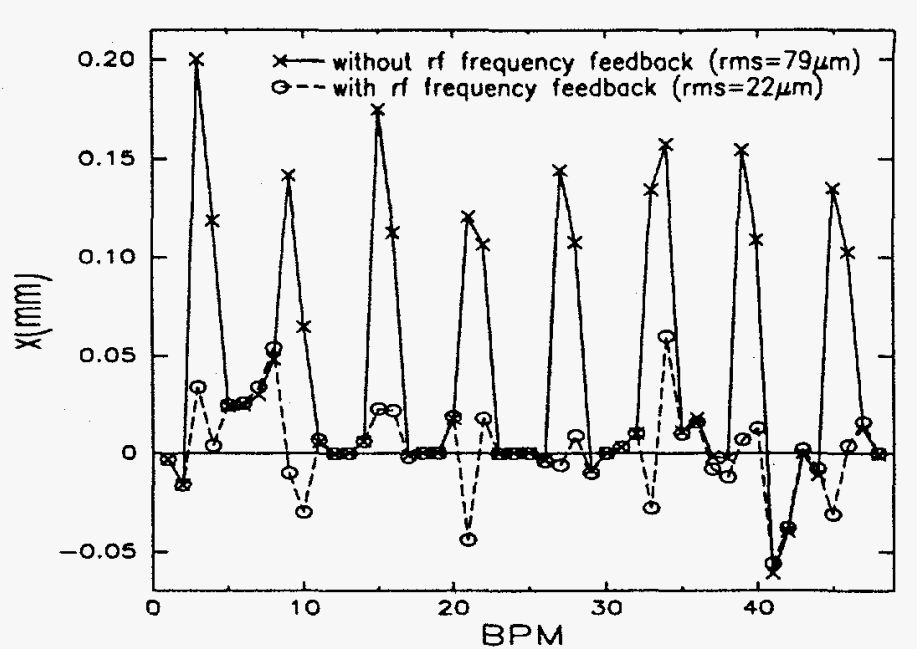

Figure. 3. Drift in horizontal orbit over several hours at the $48 \mathrm{BPMs}$ with and without rf frequency feedback. The If frequency feedback reduced the rms orbit drift by nearly a factor of four during this fill.

The $f_{r f}$ feedback is already running routinely on the NSLS UV Ring. It has run on several fills on the X-Ray Ring, and we plan to put it into regular operations soon.

\section{BPM MOTION MEASUREMENTS $[4,5]$}

As the vacuum chamber heats and expands from synchrotron radiation, the beam position monitors (BPMs) also move [6]. In each superperiod of the X-Ray Ring, a rigid vacuum chamber support near $Q 4$ (see figure 4) fixes the chamber position in all three dimensions. The chamber is free to expand longitudinally between $\mathrm{Q} 4$ and the bellows, which are located at the end of the insertion straight section. Several supports between the three-dimensional fixed point and the bellows minimize transverse horizontal and vertical motion while permitting the longitudinal expansion. Despite these supports, the large forces associated with the vacuum chamber expansion lead to significant motion of the vacuum chamber in the transverse horizontal direction in certain locations as well as small vertical motions.

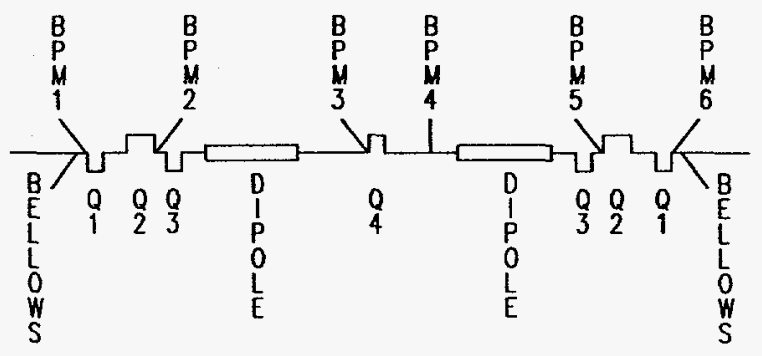

Figure. 4. This is a schematic of the layout of one of the 8 superperiods in the X-Ray Ring. The quadrupole, dipole, and BPM locations are shown.

Gauges installed at several BPMs measured the transverse movement of the vacuum chamber. Figure 5 shows 
the largest measured motion, which was transverse horizontal motion at BPM 5 in the sixth superperiod. The most motion is at BPM 5 in each superperiod, because it is just downstream of the dipole magnet (figure 4) where synchrotron radiation heating is largest. A large chamber movement (about $300 \mu \mathrm{m}$ in figure 5) occurs as the ring energy is ramped to $2.584 \mathrm{GeV}$, when the synchrotron radiation begins heating the chamber. The vacuum chamber is cooled by temperature regulated water, but even with the inlet water temperature held fixed, the outlet water temperature heats 1.8 degrees $\mathrm{C}$ from the synchrotron radiation. After the initial large motion which lasts about 10 minutes, there is a slow drift in the chamber position as the synchrotron radiation load decreases due to stored current decay.

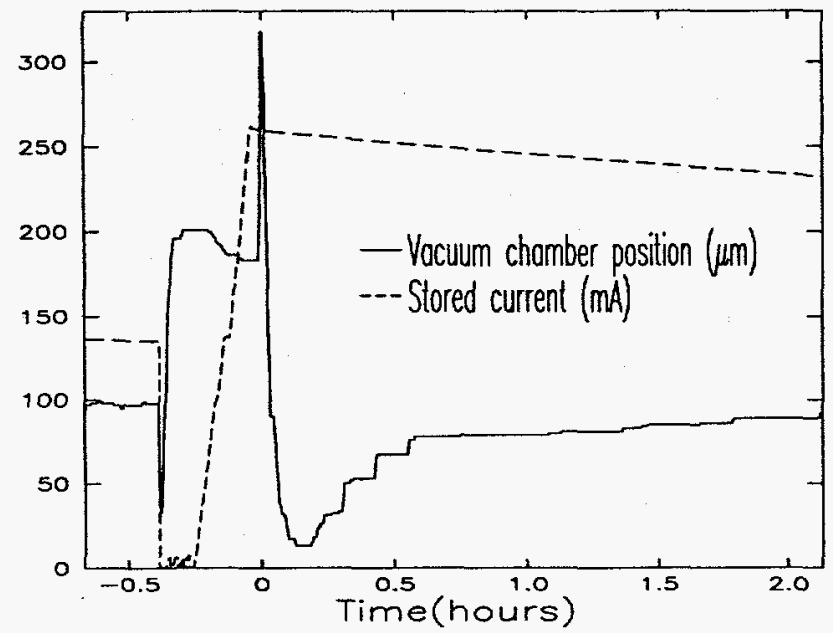

Figure. 5. This shows the horizontal motion of the vacuum chamber at BPM 5 in the sixth superperiod as the beam is dumped and refilled. Time zero is the time the ring energy was ramped from injection energy $(744 \mathrm{MeV})$ to operation energy $(2.584 \mathrm{GeV})$.

Table 2 shows the size of the initial horizontal motion (the first 5 to 10 minutes of the fill) and the subsequent drift in the vacuum chamber over the succeeding hours of the fill for the BPMs measured so far. The limit on the long-term horizontal orbit stability of the X-Ray Ring is set by this vacuum chamber motion, since the orbit motions associated with the feedback systems are much smaller. A possibility for removing this limit is compensating for the measured BPM motions by modifying the orbit feedback setpoints as the BPMs move, either in real-time or with a look-up table.

We measured the vertical motion of the vacuum chamber at the two BPMs with the largest measured horizontal motion. Both had initial vertical motions of less than 20 $\mu m$. Subsequent vertical motion over the succeeding hours of the fill was even less, and the motion at BPMs where the vacuum chamber sees less synchrotron radiation is expected to be smaller still. At this time, stabilization of the vertical orbit is limited more by the variation of BPM electronics with stored current than by BPM motion.
Table 2. The initial motion is the horizontal movement of the BPM in the first 5 to 10 minutes of the fill. The subsequent motion is the movement in the following several hours of the fill.

\begin{tabular}{|c|c|c|c|}
\hline BPM & Superperiod & $\begin{array}{c}\text { Initial } \\
\text { Motion }\end{array}$ & $\begin{array}{c}\text { Subsequent } \\
\text { Motion }\end{array}$ \\
\hline 2 & 8 & $20 \mu m$ & $<10 \mu \mathrm{m}$ \\
3 & 2 & $0 \mu \mathrm{m}$ & $0 \mu \mathrm{m}$ \\
4 & 2 & $60 \mu \mathrm{m}$ & $50 \mu \mathrm{m}$ \\
5 & 1 & $90 \mu \mathrm{m}$ & $60 \mu \mathrm{m}$ \\
5 & 6 & $300 \mu \mathrm{m}$ & $70 \mu \mathrm{m}$ \\
5 & 7 & $240 \mu \mathrm{m}$ & $85 \mu \mathrm{m}$ \\
6 & 7 & $40 \mu \mathrm{m}$ & \\
\hline
\end{tabular}

\section{CONCLUSION}

We have reduced short-term orbit fluctuations by as much as a factor of 3 by increasing the gain and bandwidth of the global harmonic feedback system. We have reduced long-term horizontal orbit drift by as much as a factor of 3.6 by using the rf frequency in the orbit feedback. We are pursuing the possibility of further reducing the horizontal orbit drift by compensating for measured horizontal movement of the vacuum chamber at the beam position monitors. Further reduction in the vertical orbit drift will be achieved when we implement recently developed BPM processing electronics, which have improved dynamic range with stored current.

\section{ACKNOWLEDGEMENTS}

The authors appreciate useful discussions with Eric Johnson, Sam Krinsky, and Peter Stefan. Yong Tang and John Smith's help with the computer code for the rf frequency feedback was appreciated. We would like to thank Bob Foxworth, Bob Harrington, Denny Klein, Ed McKenna, Susila Ramamoorthy, and George Stenby for their help with the vacuum chamber motion measurements.

\section{References}

[1] O. Singh, proceedings of the 1993 Particle Accelerator Conference, pg 2254.

[2] R. Nawrocky, to be published.

[3] E. Bozoki, A. Friedman, S. Ramamoorthy, these proceedings.

[4] A. Ropert, AIP Conference Proceedings 315, 1994, pg 69.

[5] J.A. Balmer, L.A. Welbourne, proceedings of the 1994 European Particle Acclerator Conference, pp 25612563.

[6] E. Johnson, A.M. Fauchet, X. Zhang, Correlation of Photon Beam Motion with Vacuum Chamber Cooling on the NSLS X-Ray Ring, Rev. Sci. Instrum. 63 (1), January, 1992. 


\section{DISCLAIMER}

This report was prepared as an account of work sponsored by an agency of the United States Government. Neither the United States Government nor any agency thereof, nor any of their employees, makes any warranty, express or implied, or assumes any legal liability or responsibility for the accuracy, completeness, or usefulness of any information, apparatus, product, or process disclosed, or represents that its use would not infringe privately owned rights. Reference herein to any specific commercial product, process, or service by trade name, trademark, manufac: turer, or otherwise does not necessarily constitute or imply its endorsement, recommendation, or favoring by the United States Government or any agency thereof. The views and opinions of authors expressed herein do not necessarily state or reflect those of the United States Government or any agency thereof. 


\section{DISCLAIMER}

Portions of this document may be illegible in electronic image products. Images are produced from the best available original document. 